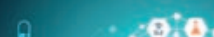

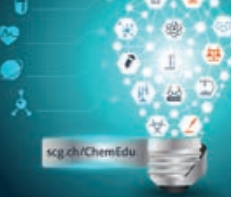 Chemical Education \\ Taste Perception: Molecular Recognition of Food Molecules
}

\section{Yvonne Westermaier*}

${ }^{*}$ Correspondence: Dr. Y. Westermaier, E-mail: yvonne.westermaier@gmail.com Pettenkofengasse 2/4, 1030 Wien, Austria.

Abstract: Molecular recognition of food molecules by ion channels and G-protein coupled receptors is the basis of taste perception. We explore the chemical nature of dietary molecules, and explore how salty, sour, sweet, bitter, and umami tastes can be explained at a molecular level.

Keywords: Chemical education · G-protein coupled receptor . Ion channel · Taste

\section{Molecular Mechanisms of Taste}

There is no accounting for taste. Yet, at every meal, we detect at least five basic taste qualities: salty, sour, sweet, bitter, and the savoury taste umami. Because taste perception is an everyday topic, the molecular mechanisms of taste can be taught both in a traditional and an active learning way, where students work in groups on different tastes as case studies. This educational column briefly summarizes the current knowledge on the molecular mechanisms of taste.

Taste perception is mediated by receptor proteins on the taste buds in the tongue: While salty and sour are detected by ion channels (Fig. 1A), sweet, bitter, and umami are recognized by G-protein coupled receptors (GPCRs) (Fig. 1B). ${ }^{[1]}$ Each taste serves a specific function in identifying components of our diet, such as small ions like $\mathrm{Na}^{+}$(salty taste), the protons of aversive acids (sour taste), carbohydrates (sweet taste), various bitter molecules, or amino acids (umami taste).

Sodium salts are mainly detected by the transmembrane epithelial sodium channel (Fig. 1A; reviewed in ref. [3]), which is voltage-insensitive, and selective for $\mathrm{Na}^{+}$over other cations. It is particularly important in the regulation of salt and water homeostasis in the kidney.

Sour-tasting acids often provoke aversion, which may have to do with the need to avoid spoiled food and maintaining acid-base homeostasis. Ingesting acids leads to a lower $\mathrm{pH}$ accelerating dental caries and provoking metabolic acidosis (ref. [3] and refs therein). Intriguingly, weak acids such as citric acid taste sourer than strong acids at comparable $\mathrm{pH}$. This is because weak acids are only partially dissociated in water, and their titratable acidity is higher than for strong acids, making titratable acidity a good predictor of sour taste intensity. ${ }^{[3,4]}$ Several channels and transporters could be sour taste receptors, but convincing experimental proofs are yet lacking. ${ }^{[1]}$

Many bitter compounds stem from toxic plant metabolites, which is why bitter taste receptors have probably evolved as a protection mechanism against the ingestion of poisonous food.
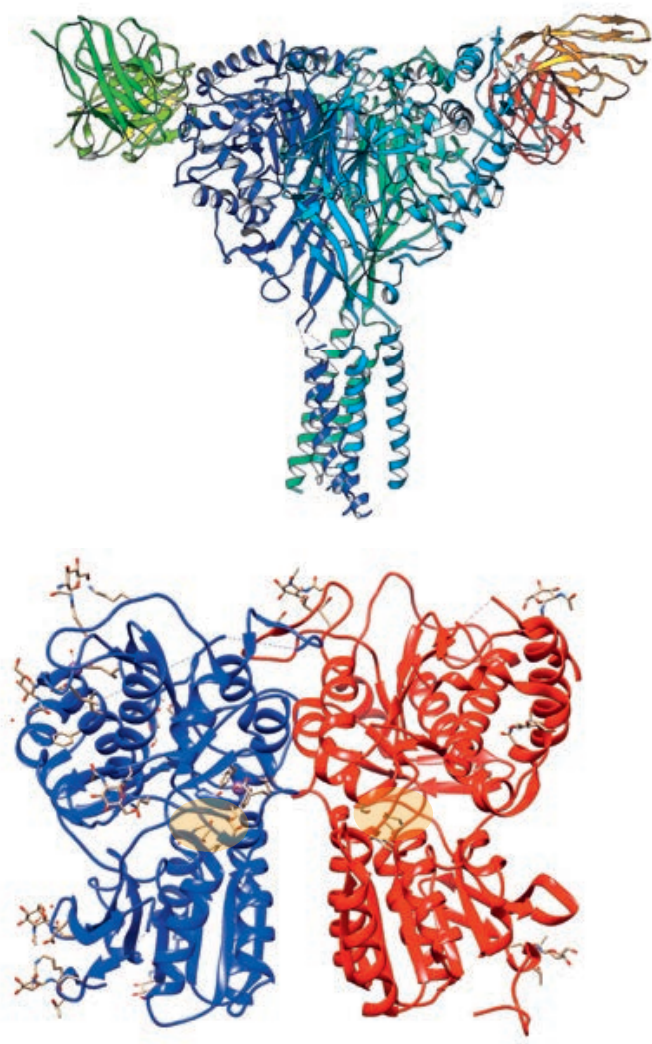

Fig. 1. Examples for $(A)$ an ion channel and $(B)$ the taste moleculebinding domain of a heterodimeric G-protein coupled receptor (GPCR). (A) shows the cryo electron microscopy structure of the membraneembedded human epithelial sodium channel that detects salts, and (B) the X-ray structure of the Japanese rice fish umami receptor with bound glutamate (yellow zone). The Figures were created with UCSF Chimera ${ }^{[2]}$ using the PDB structures $6 B Q N$ and $5 X 2 Q$, respectively. UCSF Chimera was developed by the Resource for Biocomputing, Visualization, and Informatics at the University of California, San Francisco, with support from NIH P41-GM103311.

Bitter compounds are highly diverse in terms of structure and physicochemical properties. ${ }^{[5,6]}$ As a consequence, some $25 \mathrm{hu}$ man GPCRs can bind these compounds, and individual receptors are activated by both structurally related and unrelated bitter compounds while preserving selectivity for chemical groups and even stereo-selectivity. ${ }^{[7-10]}$ The most bitter compound known to date, denatonium, is used as a synthetic bitterant to prevent inappropriate ingestion of harmful methanol or as a repellent of large animals such as deer. However, bittering agents are sometimes also intentionally ingested: Coffee and unsweetened cocoa are just two examples.

The perception of sweet and umami taste depends upon G-protein coupled receptors of class $\mathrm{C}$, which are membrane- 
embedded receptors located in the taste buds of the tongue. While both taste receptors share the same transmembrane part, in each case a unique so-called 'Venus flytrap' (VFT) domain binds molecules on the cell surface and mediates taste recognition (Fig. 1B).[11]

Carbohydrates owe their taste to the multiple H-bond interactions they form with their respective receptor. From a chemical point of view, monosaccharides have a rather similar structure, and it has been puzzling why some taste sweeter than others. For instance, the sweetness of a particular sugar does neither depend upon its solubility nor its number of glucose rings. On a relative sweetness scale with sucrose sweetness set to 1 , mannose is tasteless, glucose has a sweetness of 0.47 , and fructose $1.13,{ }^{[12]}$ hinting at a role of minor stereochemistry differences in sweetness perception. Intrigued by this question, Ricci and colleagues performed neutron diffraction experiments on three monosaccharides (fructose, glucose, and mannose) and the disaccharide trehalose in water. ${ }^{[13]}$ For example, fructose can be found in apples and pears, glucose in stark sugar, mannose in strawberries, and trehalose helps oysters to prevent ice formation. Neutron diffraction is powerful for investigating the hydration of small molecules, as it detects correlations between oxygens and hydrogens particularly well. While the overall hydration shell is not informative, the length of $\mathrm{H}$-bonds $\left(\mathrm{r}_{\mathrm{HB}}\right)$ between the sugars and the surrounding water determines sweetness (Fig. 2).

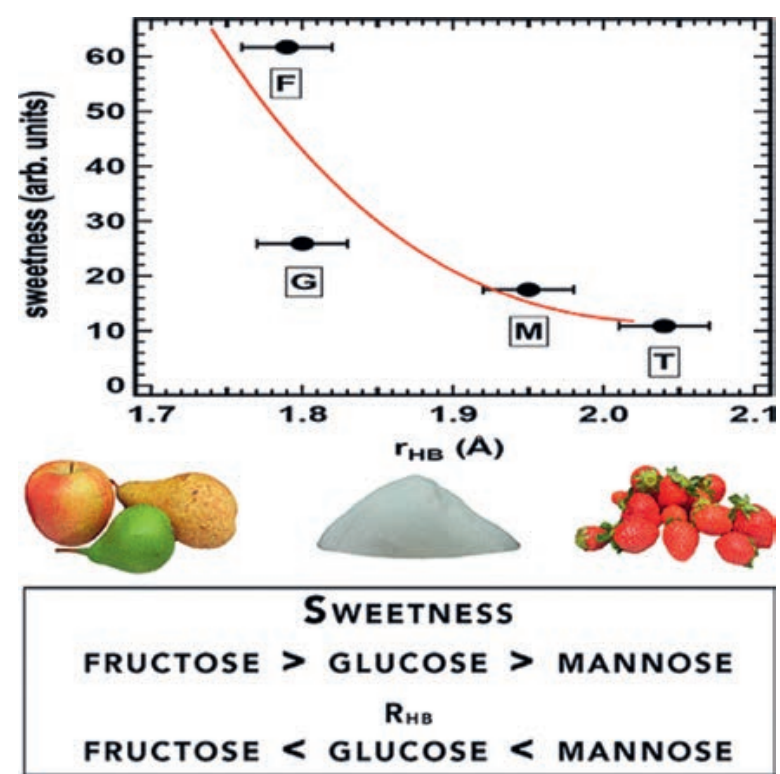

Fig. 2. Sweetness as a function of the length of the $\mathrm{H}$-bond ( $\mathrm{rHB}$ ) of different sugars with surrounding water molecules as determined by neutron diffraction. F stands for fructose, G for glucose, M for mannose, and $\mathrm{T}$ for trehalose. Graphical abstract reprinted with permission from Bruni et al. ${ }^{[13]}$ Copyright (2018) American Chemical Society.

Indeed, each monosaccharide forms H-bonds of different lengths: sweeter-tasting sugars form tighter, stronger bonds with the solvent and likely also with the GPCR. It is therefore not only the number and spatial arrangement of $\mathrm{H}$-bond donors and acceptors within a sugar that matters for sweet taste but above all the H-bonding strength. Additionally, the small stereochemical differences among the different sugars investigated determine relative polarity changes, which may in turn influence $\mathrm{H}$-bonding strength.

The only disaccharide included in the study, trehalose, contains two glucose rings, yet features a much lower sweetness than the monosaccharides. As pointed out by the authors, this means probably that one glucose ring is sufficient for triggering the sweet taste signal transduction from the GPCR to the brain, and that binding to the receptor pocket requires the molecule to bend to fit.[13]

Umami is perhaps the least known taste, although it is frequently encountered in Asian cuisine. It stems from the amino acid glutamate and is enhanced by ribonucleotides. Li and colleagues ${ }^{[11]}$ have recently discovered that glutamate is recognized by four amino acids inside the GPCR's VFT domain. The tasteenhancing ribonucleotides are however detected by another four amino acids in the opening of the same domain. It is hypothesized that the binding of such regulatory molecules near glutamate contributes to maintaining the taste molecule-bound, closed conformation.

In summary, the chemistry of taste involves the molecular recognition of food molecules by ion channels or GPCRs. Whether a molecule binds to a channel or a GPCR depends on its capacity to form strong, favourable interactions. Particularly, the sweetness of sugar is determined by the length of its H-bonds with surrounding water molecules, and presumably also its interaction with a receptor.

Received: April 25, 2021

[1] A. A. Bachmanov, G. K. Beauchamp, Ann. Rev Nutr. 2007, 27, 389. https://doi.org/10.1146/annurev.nutr.26.061505.111329

[2] E. F. Pettersen, T. D. Goddard, C. C. Huang, G. S. Couch, D. M Greenblatt, E. C. Meng, T. E. Ferrin, J. Comput. Chem. 2004, 25, 1605, https://doi.org/10.1002/jcc.20084

[3] S. D. Munger, in 'Chemosensory Transduction', Eds. F. Zufall, S. D. Munger, Academic Press, 2016, 287, https://doi.org/10.1016/B978-0-12-801694-7.00016-0

[4] E. R. Da Conceitao Neta, S. D. Johanningsmeier, R. F. McFeeters, J. Food Sci. 2007, 72, R33, https://doi.org/10.1111/j.1750-3841.2007.00282.x

[5] M. Behrens, W. Meyerhof, Cell Mol. Life Sci. 2006, 63, 1501, https://doi.org/10.1007/s00018-006-6113-8

[6] W. Meyerhof, S. Born, A. Brockhoff, M. Behrens, Flavour Frag. J. 2011, 26, 260, https://doi.org/10.1002/ffj.2041

[7] A. Brockhoff, M. Behrens, A. Massarotti, G. Appendino, W. Meyerhof, J. Agric. Food Chem. 2007, 55, 6236, https://doi.org/10.1021/jf070503p

[8] B. Bufe, P. A. Breslin, C. Kuhn, D. R. Reed, C. D. Tharp, J. P. Slack, U. K. Kim, D. Drayna, W. Meyerhof, Curr. Biol. 2005, 15, 322, https://doi.org/10.1016/j.cub.2005.01.047

[9] B. Bufe, T. Hofmann, D. Krautwurst, J. D. Raguse, W. Meyerhof, Nat. Genet. 2002, 32, 397, https://doi.org/10.1038/ng1014

[10] A. Wiener, M. Shudler, A. Levit, M. Y. Niv, Nucl. Acids. Res. 2012, 40 D413, https://doi.org/10.1093/nar/gkr755

[11] F. Zhang, B. Klebansky, R. M. Fine, H. Xu, A. Pronin, H. Liu, C. Tachdijan, X. Li, Proc. Nat. Acad. Sci. 2008, 105, 20930, https://doi.org/10.1073/pnas.0810174106

[12] M.-O. Portmann, G. Birch, J. Sci. Food Agricult. 1995, 69, 275, https://doi.org/10.1002/jsfa.2740690303

[13] F. Bruni, C. Di Mino, S. Imberti, S. E McLain, N. H. Rhys, M. A. Ricci, J. Phys. Chem. Lett. 2018, 9, 3667, https://doi.org/10.1021/acs.jpclett.8b01280 